\title{
When $e$-th Roots Become Easier Than Factoring
}

\author{
Antoine Joux ${ }^{1, \star}$, David Naccache ${ }^{2}$, and Emmanuel Thomé ${ }^{3}$ \\ ${ }^{1}$ DGA and Université de Versailles, UVSQ PRISM 45 avenue des États-Unis, \\ F-78035 Versailles CEDEX, France \\ antoine.joux@m $4 \mathrm{x}$.org \\ 2 École normale supérieure, Équipe de cryptographie, 45 rue d’Ulm, \\ F-75230 Paris CEDEX 05, France \\ david.naccache@ens.fr \\ 3 INRIA Lorraine, LORIA, CACAO - bâtiment A, 615 rue du Jardin botanique, \\ F-54602 Villiers-lès-Nancy CEDEX, France \\ emmanuel.thome@normalesup.org
}

\begin{abstract}
We show that computing $e$-th roots modulo $n$ is easier than factoring $n$ with currently known methods, given subexponential access to an oracle outputting the roots of numbers of the form $x_{i}+c$.

Here $c$ is fixed and $x_{i}$ denotes small integers of the attacker's choosing. The attack comes in two flavors:

- A first version is illustrated here by producing selective roots of the form $x_{i}+c$ in $L_{n}\left(\frac{1}{3}, \sqrt[3]{\frac{32}{9}}\right)$. This matches the special number field sieve's (SNFS) complexity.

- A second variant computes arbitrary e-th roots in $L_{n}\left(\frac{1}{3}, \gamma\right)$ after a subexponential number of oracle queries. The constant $\gamma$ depends on the type of oracle used.

This addresses in particular the One More RSA Inversion problem, where the $e$-th root oracle is not restricted to numbers of a special form. The aforementioned constant $\gamma$ is then $\sqrt[3]{\frac{32}{9}}$.

Constraining the oracle to roots of the form $\sqrt[e]{x_{i}+c} \bmod n$ increases $\gamma$.

Both methods are faster than factoring $n$ using the GNFS $\left(L_{n}\left(\frac{1}{3}, \sqrt[3]{\frac{64}{9}}\right)\right)$.

This sheds additional light on RSA's malleability in general and on RSA's resistance to affine forgeries in particular - a problem known to be polynomial for $x_{i}>\sqrt[3]{n}$, but for which no algorithm faster than factoring was known before this work.
\end{abstract}

Keywords: RSA, factoring, NFS, roots.

\section{Introduction}

The RSA cryptosystem [17] is commonly used for providing privacy and authenticity of digital data. A very common historical practice for signing with RSA

* Work partially supported by DGA research grant 05.34.058.

K. Kurosawa (Ed.): ASIACRYPT 2007, LNCS 4833, pp. 13-28, 2007.

(C) International Association for Cryptology Research 2007 
was to first hash the message, add a padding pattern $c$ and then raise the result to the power of the decryption exponent. This paradigm is the basis of numerous standards such as PKCS\#1 v1.5 [18].

Let $n$ and $e$ denote usual RSA public parameters (with $\left.\left\lceil\log _{2} n\right\rceil=N\right) \sqrt{1}$.

In this paper we explore RSA signatures with a fixed $c$ but without the hash function, i.e. modular roots of the form:

$$
\sqrt[e]{c+x} \bmod n
$$

We call such numbers affine modular roots (AMRs).

A thread of publications 15/7/10/14/4/3 stretching over a decade progressively established that given access to an AMR-oracle, new AMRs could be forged in polynomial complexity for $x>\sqrt[3]{n}$.

No strategies faster than factoring $n$ are known for $x<\sqrt[3]{n}-$ a case tackled here at the cost of subexponential complexity. The main novelty in this paper is that, while subexponential, our method forges new AMRs for arbitrarily small $x$ (down to $x<\sqrt[\epsilon]{n}, \forall \epsilon>0$ ) faster than factoring $n$.

Moreover, we show that access to an $e$-th root oracle (in particular, an AMRoracle) even allows to compute arbitrary $e$-th roots faster than factoring $n$. Here, the arbitrary $e$-th root to be computed is not known before all oracle queries have been completed.

We achieve this by tweaking the quadratic sieve (QS) and the number field sieve (NFS) factoring algorithms.

The Results. Denoting $L_{n}(\alpha, c)=\exp \left(c(1+o(1))(\log n)^{\alpha}(\log \log n)^{1-\alpha}\right)$, we show that:

- Using a QS-like algorithm, new AMRs can be computed in $L_{\frac{n}{x}}\left(\frac{1}{2}, 1\right)$ instead of the $L_{n}\left(\frac{1}{2}, 1\right)$ required for QS-factoring $n$.

- Using an NFs-like approach, we selectively produce new AMRs in $L_{n}\left(\frac{1}{3}, \sqrt[3]{\frac{32}{9}}\right)$.

This matches the special number field sieve's (SNFS) complexity which is substantially lower than the $L_{n}\left(\frac{1}{3}, \sqrt[3]{\frac{64}{9}}\right)$ required to GNFS-factor $n$.

Our experimental results for $N=512$ and a recent SNFS-factoring record 2 , clearly underline the insecurity of affine-padding RSA.

- We present a procedure for computing arbitrary $e$-th roots in $L_{n}\left(\frac{1}{3}, \sqrt[3]{\frac{32}{9}} \simeq\right.$ 1.53), requiring a general (not only AMR) $e$-th root oracle.

A more practical variant with a slightly higher complexity $L_{n}\left(\frac{1}{3}, 1.58\right)$ was used in the experiments reported in this paper.

- Finally, a last variant allows the computation of arbitrary $e$-th roots given access to an AMR-oracle with complexity $L_{n}\left(\frac{1}{3}, \sqrt[3]{6}\right)$. To date, we could not make this variant practical.

\footnotetext{
${ }^{1}$ Throughout this paper, we will frequently denote by $|x|$ the bitlength of $x$.
}

${ }^{2}$ [1, factoring a 1039-bit number using $\simeq 95$ Pentium-D-years at $3 \mathrm{GHz}$. 
Our algorithms rely on an extension of Montgomery's square root algorithm for the number field sieve [16. If one avoids this algorithm, alternative variants exist with claim a higher complexity $\left(L_{n}\left(\frac{1}{3}, \sqrt[3]{\frac{9}{2}}\right)\right)$.

\section{The Strategy - A General Outline}

For the sake of simplicity assume that $|x|=\frac{N}{4}$ (generalization to smaller $x$ sizes is straightforward). We start by writing $c$, as a modular ratid3:

$$
c=\frac{a}{b} \bmod n \text { where }|a|=(1-s) N \text { and }|b|=s N
$$

for some $0<s<1$ that will be determined later.

Noting that $c+x=\frac{a+x b}{b} \bmod n$, it is easy to derive an index calculus attack 4 as in 6 6 on numbers of the form $a+x b$, that we expect to be smooth with respect to some factor base $\mathcal{B}$. We can ascertain that $a+x b$ is partially smooth by applying a special- $q$ strategy. Two options are possible: Either choose different partial products of size $\frac{N}{4}$ of primes belonging to $\mathcal{B}$ (denote these partial products $u_{i}$ ) and sieve on $x_{i}$ values such that $x_{i}=-c \bmod u_{i}$ or, select as special- $q$ primes of size $\frac{N}{4}$ and use them as the $u_{i}$ in the first option. From an asymptotic standpoint, the two approaches are equivalent. In practice, the first approach can produce any given equation more than once and thus require extra bookkeeping. As for the second approach, each special- $q$ requires one extra equation to cancel out, thereby resulting in a larger system of equations.

It remains to optimize $s$. To maximize the smoothness odds of $a+x b$ we require that $|a|=|x b|$ hence:

$$
(1-s) N=|a|=|x b|=|x|+|b|=\frac{N}{4}+|b|=\frac{N}{4}+s N \quad \Rightarrow \quad s=\frac{3}{8}
$$

In other words, we need to find multiplicative relations between numbers of size $\frac{5 N}{8}$ divisible, by construction, by smooth factors of size $\frac{N}{4}$. All in all this amounts to chasing smooth numbers of size $\frac{3 N}{8}$ which is easier than QS-factoring $n$ (identical task for numbers of size $\frac{N}{2}=\frac{4 N}{8}$ ).

More generally, when $x$ is an $\frac{N}{t}$ bit number, the job boils-down to finding smooth numbers of size $\frac{N(t-1)}{2 t}$ i.e. QS-factoring $\frac{N(t-1)}{t}$ bit RSA moduli.

Hence, the presented strategy approaches the QS's complexity as $t$ grows, while remaining below the QS's complexity curve6.

${ }^{3}$ E.g. Using a continued fraction algorithm.

4 Treat $b$ as an extra element of the factor base, together with the primes in the basis to account for the denominator in the equations.

${ }^{5}$ In [6] the signing oracle is used to compute $e$-th roots whose combination allows to compute new $e$-th roots of factor-base elements.

${ }^{6}$ To sieve, it suffices to set $x_{i}=-c \bmod u_{i}$ and consider successively $a\left(x_{i}+j u_{i}\right)+b$ for $j=1,2, \ldots$ (note that this will pollute a logarithmic number of bits in $c$ ). 
Given that the quadratic-sieve is not the fastest factoring strategy for usualsize RSA moduli, the extension of the above strategy to the NFS is a natural question (that this paper answers positively).

NFS algorithms work by exhibiting relations between objects in two different "worlds". In some cases, we have a single number field and consider relations between integers and elements in that field. In other cases, there are two number fields. Nonetheless, with both approaches, there are two sides to consider. In this paper, the AMR-oracle is going to replace one of the two sides. Consequently, our setting is that of a single-sided NFS. This turns out to greatly improve the smoothness probability and hence the algorithm's efficiency.

We start by selecting a parameter $d$ and finding a polynomial $f$ of degree $d$ having sufficiently small coefficients such that $f(c) \equiv 0 \bmod n$. Without loss of generality, we may assume that $f$ is irreducible over $\mathbb{Q}$. Indeed, if $f=f_{1} \times$ $f_{2}$, either $\operatorname{gcd}\left(f_{1}(c), n\right)$ is a non-trivial factor of $n$, or we can use the (smaller) polynomial $f_{1}$ instead of $f$.

Once $f$ is chosen, we construct the number field $K=\mathbb{Q}[\alpha]$ where $\alpha$ is a root of $f$ over $\mathbb{Q}$. We now proceed as in the NFS and given integers $x$, we construct elements $\alpha+x \in \mathbb{Q}[\alpha]$ with smooth norm over some factor base $\mathcal{B}$. We recall that the norm of $\alpha+x$ is the absolute value of $f(-x)$. Note a major difference with NFS-factoring: indeed, we only need to smooth a single $\alpha+x$ for each candidate $x$ as there is no second (or rational) side to smooth in addition. Instead, the second side is given for free by the AMR-oracle for the number corresponding to $\alpha+x$, i.e. for $c+x$. When the norm is smooth, we can decompose $\alpha+x$ into a product of ideals of small norm in the ring of integers $\mathcal{O}_{K}$ of $K=\mathbb{Q}[\alpha]$.

Once enough smooth elements are found, we write them down as rows in a matrix where each row contains the valuation of the corresponding $\alpha+x$ at each prime ideal occurring in its decomposition. We also add to each row enough character maps in order to account for the existence of units in the number field.

Then, using a sparse linear algebra algorithm, we find a linear combination of rows equal to zero modulo $e$. This allows us to write an $e$-th power in $\mathbb{Q}[\alpha]$ as a product of $\alpha+x_{i}$.

The final step computes the actual $e$-th root of this $e$-th power. This yields a multiplicative relation between AMRs corresponding to the $\alpha+x_{i}$ used in the relation. Thus, querying all these values but one yields a new AMR for the missing value. The $e$-th root can be computed in a way very similar to the NFs' square root computation phase.

Alternatively, the final step can be replaced by a more involved strategy. Namely, combining the $e$-th root computation with a descent procedure very similar to the individual logarithm step of discrete logarithm computations with the NFS. This enables the calculation of $e$-th roots of arbitrary values, i.e. not restricted to the form $c+x$, by making a small number of additional queries of the restricted form $c+x$. This option is presented in Section 4 . 


\section{A Detailed Step-by-Step Description}

\subsection{Polynomial Construction}

Given a target degree $d$ we first need to construct a polynomial $f$ irreducible over $\mathbb{Q}$. $f$ will then be used to define the number field $K=\mathbb{Q}[\alpha]$. The two important constraints on $f$ are that its coefficients should be small and that we must have $f(c)=0 \bmod n$. Since we want to minimize the average norm $f(-x)$ of numbers $\alpha+x$, it is a good idea to use a skewed polynomial. More precisely, assume that $B$ bounds the absolute value of $x$, then we want to choose a bound $C$ such that the coefficient of degree $i$ in $f$ has absolute value smaller than $\frac{C}{B^{i}}$. Assuming 7 that $B^{d(d+1)}<n$, we choose $C=\sqrt[d+1]{n} B^{d / 2}$ and the polynomial $f$ can be constructed by reducing the lattice generated by the columns of the $(d+1) \times(d+1)$ matrix

$$
L=\left(\begin{array}{cccc}
\Lambda & \cdots & \Lambda c^{d} & \Lambda n \\
1 & 0 & 0 \\
\ddots & & \vdots \\
0 & B^{d} & 0
\end{array}\right)
$$

where $\Lambda$ is a sufficiently large constant to guarantee that any short vector in the lattice has zero in its first coordinate. Such a short vector can be easily interpreted as a polynomial by reading the coefficient of $x^{i}$ in row $i+2$ (the coefficient should be re-normalized by a division by $B^{i}$ ). This polynomial clearly has $c$ as a root modulo $n$. Moreover, when evaluating the polynomial at $x$ smaller than $B$ (in absolute value) we see that each term is bounded by the corresponding value in the initial short vector.

Since the determinant of $L$ is $n B^{d(d+1) / 2}$, we expect short-vector coefficients to be of size

$$
C=\sqrt[d+1]{n} B^{\frac{d}{2}} 2^{\frac{d}{4}}
$$

\subsection{Sieving}

From a sieving standpoint, there is an essential difference between our algorithm and the NFS. Indeed, our sieving has a single degree of freedom instead of two. More precisely, instead of scanning numbers of the form $a \alpha+b$ for a fixed $\alpha$ and arbitrary pairs of small $\{a, b\}$, we need to examine numbers of the form $\alpha+x$.

Luckily, the bound on $x$ is large enough to compensate the absence of the second degree of freedom but this restricts our sieving technique options. Indeed, we cannot use a lattice sieve strategy and have to rely instead on a straightforward sieve-by-line algorithm. To avoid using large numbers while sieving over $x$, we used a special- $q$ approach: for each special- $q$ prime ideal $\langle q, \alpha-r\rangle$, we considered the algebraic integers $\alpha+(q x-r)$, with $x \in\left[-\frac{S}{q},+\frac{S}{q}\right]$.

\footnotetext{
7 This is necessary to avoid finding zero for high degree coefficients; of course, where necessary, we can always lower $B$ in this construction and sieve over a smaller $x$ range (as long as enough equations are found.).
} 


\subsection{Linear Algebra and Characters}

Depending on the size of $e$, one may either use Lanczos/Wiedeman or block Lanczos/Wiedeman approach. If $e$ is large enough, no self-orthogonal vector appears (unless we are extremely unlucky) and the simple approach succeeds. For smaller $e$, a block approach is required (the block size $2 \leq z \leq 32$ varies with $e$ and is a bit lesser when $e=3$ ).

When linear algebra is performed directly on the sieving phase's output, the method yields a multiplicative relation between ideals of the form:

$$
\prod_{i}\left\langle\alpha+x_{i}\right\rangle^{\mu_{i}}=\left(\prod_{j} \mathfrak{p}_{j}^{\nu_{j}}\right)^{e}
$$

Such a relation, however, is insufficient to ensure that the product $\prod_{i}\left(\alpha+x_{i}\right)^{\mu_{i}}$ is an $e$-th power in $K$. Obstructions may arise from the $e$-part of the ideal class group of $\mathcal{O}_{K}$, as well as from the quotient of the unit group $\mathcal{O}_{K}^{*} /\left(\mathcal{O}_{K}^{*}\right)^{e}$. To annihilate these obstructions we have to add characters. We require that:

$$
\chi\left(\prod_{i}\left(\alpha+x_{i}\right)^{\mu_{i}}\right)=\sum_{i} \mu_{i} \chi\left(\alpha+x_{i}\right)
$$

vanishes, for several (additive) character maps $\chi: K^{*} \rightarrow \mathbb{F}_{e}$. We have the following choices for character maps:

- In [19, an approximation of the e-adic logarithm is used. Such characters are easy to compute but might fail to account for the full obstruction, as they cover at most the obstruction stemming from the unit group. Should $e$ ramify in $\mathcal{O}_{K}$, or $e \mathcal{O}_{K}$ be divisible by a prime ideal belonging to the factor base, technicalities occur but do not prevent from using these characters.

- It is also possible to follow the classical approach used for NFS-factoring 3 i.e. test for powers modulo primes congruent to $1 \bmod e$. The number of characters accessible thereby is infinite. To map these multiplicative characters to additive ones, a discrete logarithm modulo $e$ must be solved, which is trivial for small $e$. For larger $e$ values (where this might be a problem) heuristic arguments indicate that characters of the first kind would suffice anyway [19].

A typical drawback of characters is that they add a dense part to the relation matrix, which might cause a slight performance penalty. In the particular case we are interested in (just as in NFS-factoring) it is possible to perform the linear algebra without the character columns, produce several row dependencies and do a second reduction to recombine these dependencies into dependencies with vanishing characters.

If we elect to adopt the latter idea it becomes particularly advisable to use block algorithms for the linear algebra, since these algorithms output several vectors of the null-space simultaneously. 
The linear algebra step also gives us the opportunity to check that $K$ 's class number is co-prime to $e$ (to avoid possible technical problems infra). We do so by checking that the rank of the relation matrix is not abnormally low modulo $e$. This extra check is achieved in the same complexity and is therefore ignored hereafter. Moreover, when $e$ is a large prime, we do not need to test anything, since the probability that $e$ divides the class number is negligible.

\subsection{Root Extraction}

The linear algebra stage yields a product of algebraic integers $\pi=\prod\left(\alpha+x_{i}\right)^{\mu_{i}}$ which is known to be an $e$-th power in $K$ since $\chi(\pi)=0$ for satisfyingly many characters $\chi$. This allows us to compute an $e$-th root in $\mathbb{Z}_{n}$ for any $c+x_{i^{\prime}}$, as long as the corresponding exponent $\mu_{i^{\prime}} \neq 0 \bmod e$. To do so, we first have to raise $\pi$ to the power of $\mu_{i^{\prime}}^{-1} \bmod e$. In other words, we can assume without loss of generality that $\mu_{i^{\prime}}=1$.

When $e$ is small, the computation of the $e$-th root of $\pi$ can be done using a straightforward generalization of Montgomery's square root algorithm [16].

Once the $e$-th root $R(\alpha)$ is computed, we have:

$$
\begin{aligned}
\left(c+x_{i^{\prime}}\right) \prod_{i \neq i^{\prime}}\left(c+x_{i}\right)^{\mu_{i}} & =R(c)^{e} \bmod n, \\
\text { i.e.: } \quad\left(c+x_{i^{\prime}}\right)^{d} & =R(c) \prod_{i \neq i^{\prime}}\left(c+x_{i}\right)^{-\mu_{i}} \bmod n .
\end{aligned}
$$

One might question the applicability of Montgomery's algorithm to very large values of $e$. Our computations in appendix A indicate that $e=65,537$ is achievable with no difficulty and tests up to $e \simeq 10^{15}$ were conducted successfully. These results lead us to infer that this approach is practical at least for our range of interest.

However, should this strategy become difficult for larger $e$, a different (more expensive) approach might be used: replace the sparse linear algebra modulo $e$ by exact Gaussian elimination or Hermite normal form and find relations expressing each ideal as a product (quotient) of smooth elements. This associates to each ideal a projection 8 in $\mathbb{Z}_{n}$ and also its $e$-th root. The drawbacks are higher memory requirements and a higher exponent in the linear algebra's complexity.

\subsection{Complexity Analysis}

Our complexity analysis closely follows the NFS's one. Let $w$ denote the linear algebra's exponent. We write the degree $d$, the sieving range $[-S,+S]$ and the factor base bound $B$ as:

$$
d=\delta \times \sqrt[3]{\frac{\log n}{\log \log n}}, \quad S=L_{n}\left(\frac{1}{3}, w \beta\right) \text { and } B=L_{n}\left(\frac{1}{3}, \beta\right) .
$$

\footnotetext{
${ }^{8}$ In theory, such a projection can be defined rigorously using the Hilbert class field of the number field used. Indeed, in the Hilbert class field, all ideals are principal and sending a generator to $\mathbb{Z}_{n}$ is easy; however, since the degree of the Hilbert class field is extremely large, it cannot be used in practice.
} 
This particular choice of $S$ and $B$ ensures that the sieving step (which costs $S$ ) and the linear algebra step (which $\operatorname{costs} B^{w}$ ) are balanced.

Using the lattice-based construction, the coefficients of $f$ have average size $A=\sqrt[d]{n}=L_{n}\left(\frac{2}{3}, \frac{1}{\delta}\right)$. By choosing a skewed $f$, we find that the size of $f(x)$ for $x \in[-S,+S]$ is:

$$
A \times S^{d / 2}=L_{n}\left(\frac{2}{3}, \frac{1}{\delta}+\frac{w}{2} \delta \beta\right)
$$

The probability that $f(x)$ is $B$-smooth is $L_{n}\left(\frac{1}{3},-\pi\right)$ with $\pi=\frac{1}{3}\left(\frac{1}{\delta \beta}+\frac{w}{2} \delta\right)$.

To get enough smooth relations, we need to ensure that $w \beta-\pi=\beta$.

For $w=2$, these equations lead to the choice $\left\{\delta=\sqrt[3]{\frac{2}{3}}, \beta=\sqrt[3]{\frac{4}{9}}\right\}$. As a consequence, the complexity of the sieving and linear algebra steps put together is $L_{n}\left(\frac{1}{3}, 2 \beta\right)=L_{n}\left(\frac{1}{3}, \sqrt[3]{\frac{32}{9}}\right)$. This is equal to the complexity the SNFs factoring algorithm which applies to a restricted class of numbers 12 .

Another very important parameter is the number of AMR-oracle queries, which is subexponential but significatively smaller than the algorithm's runtime. This number of queries is $L_{n}\left(\frac{1}{3}, \beta\right)=L_{n}\left(\frac{1}{3}, \sqrt[3]{\frac{4}{9}}\right)$.

The alternative using integer linear algebra mentioned above yields a complexity of:

$$
L_{n}\left(\frac{1}{3}, \sqrt[3]{\frac{2 w^{4}}{9(w-1)^{2}}}\right)
$$

The case $w=3$ gives $L_{n}\left(\frac{1}{3}, \sqrt[3]{\frac{9}{2}} \simeq 1.65\right)$. Note that according to 899 , the integer linear algebra can be done with exponent $w=2.5$, which yields $L_{n}\left(\frac{1}{3}, \sqrt[3]{\frac{625}{162}} \simeq 1.57\right)$. However this approach requires asymptotically fast matrix multiplication techniques which might prove too cumbersome for cryptographic applications.

As our algorithms are subexponential, the assessment of their experimental behavior is essential. We hence implemented them and actually forged a 512-bit AMr. Details are given in Appendix A.

Open Problem - Potential Improvements: When the number of fixed pad bits is small enough, the possible sieving range of $x$ when sieving over $c+x$ (or $\alpha+x)$ may be too larg 9 .

Under such circumstances, we get some additional freedom when constructing $f$. Indeed, we may replace $c$ by some $c^{\prime} \simeq c$, thereby reducing the sieving range. Clearly, amongst all possible $c^{\prime}$ values some yield $f^{\prime}$-s whose coefficients are smaller than average.

We could not find any efficient way of taking advantage of this extra freedom to build better polynomials and further reduce the attack's complexity.

${ }^{9} \mathrm{Cf}$. To the related footnote in section 3.1 


\section{Attacking the One More RSA Inversion Problem}

Up to now, we have obtained either an AMR-forgery or an adaptive chosen ciphertext attack (CCA2) on plain RSA. In this section, we extend the attack to obtain a non adaptive chosen ciphertext attack (CCA1) on plain RSA. Equivalently, we attack the One More RsA Inversion Problem, proposed by Bellare et al., in [2]. Again, while subexponential, this attack is faster than GNFS-factoring $n$. In the context of the One More RSA Problem it is not really meaningful to assume that the initial RSA queries have a special form, thus we grant the attacker access to an unlimited $e$-th root oracle during the first phase of the attack.

Once the restriction on oracle queries is lifted, we are no longer constrained to use polynomials with a prescribed root $P$. Moreover, we are no longer limited to a single dimensional sieve, but can use a classical NFS sieve with two degrees of freedom, using a lattice sieving technique. This does not change the asymptotic complexity but allows us to reuse existing fast sieving code more easily. Not being restricted to a prescribed root, we may use any polynomial of our choice. Despite this clear gain, to solve the One More RSA Inversion Problem and become nonadaptive, we need to devise an algorithm allowing us to compute the $e$-th root of an arbitrary number without any additional oracle queries. This requires a new descent procedure since the technique sketched at the end of Section 2 requires additional oracle queries. Looking at similar problems arising in the individual discrete logarithm phase of discrete logarithms computations, we see that such a non adaptive descent can be done by alternating between two NFs sides. Thus, we need to introduce a second side into our algorithm. While, at a first glance, this seems to void our single-sided NFS complexity improvement, it turns out that this intuitive perception is false since we can initially do the single sided NFS separately for both sides.

The addition of a second side entails a complication for the descent, however. To achieve the announced complexity, the initial factor base bound is set to $L_{n}\left(\frac{1}{3}, \sqrt[3]{\frac{4}{9}}\right)$. This is well below the $L_{n}\left(\frac{1}{3}, \sqrt[3]{\frac{8}{9}}\right)$ encountered when computing discrete logarithms. This implies that the descent procedure has to descend below what is done for computing discrete logarithms. While the impact on the overall complexity is not visible, this is a clear practical concern. To compensate for this fact, we add an intermediate phase in our algorithm in order to enlarge the factor base from $L_{n}\left(\frac{1}{3}, \sqrt[3]{\frac{4}{9}}\right)$ to $L_{n}\left(\frac{1}{3}, \sqrt[3]{\frac{8}{9}}\right)$.

\subsection{The Inversion Algorithm}

Step 0 - Setup. We first set up on the algebraic side a number field $K=\mathbb{Q}(\alpha)$ defined by a polynomial equation $f(\alpha)=0$. The easiest (though not unique) choice for the second side is a rational side given by a polynomial $g$ such that $f$ and $g$ share a common root $P$ modulo $n$. The classical base- $m$ technique can be used for this purpose.

We denote by $\rho$ the rational root of $g$ (we have $\rho=m$ if $g$ is monic). 
Step 1 - Precomputation. The factor base $\mathcal{F}$ on the algebraic side consists of ideals of norm bounded by $B \simeq L_{n}\left(\frac{1}{3}, \sqrt[3]{\frac{4}{9}}\right)$. By sieving, we obtain coefficient pairs $\{x, y\}$ yielding relations of the form:

$$
(x-y \alpha)=\prod_{\mathfrak{p} \in \mathcal{F}} \mathfrak{p}^{m_{\mathfrak{p}}}, \quad \text { and } \quad \chi(x-y \alpha)=\left(\lambda_{k}\right)_{k=1, \ldots, c}
$$

where $\chi$ is a character map onto $\mathbb{F}_{e}^{c}$, for some arbitrary dimension $c$. We concatenate the coefficients $\left(m_{\mathfrak{p}}\right)$ and $\lambda_{k}$ to form the rows of a matrix $M$.

Step 2 - Factor Base Extension. The extended factor base $\mathcal{F}^{\prime}$ consists of ideals of norm bounded by $B^{\prime} \simeq L_{n}\left(\frac{1}{3}, \sqrt[3]{\frac{8}{9}}\right)$. We sieve on the algebraic side only, using each additional prime ideal that we want to add as a special- $q$. We ask for a single relation between this prime ideal and the smaller ones.

Step 3 - Oracle Queries. We query the oracle for the $e$-th root of the numbers $x-y P$ for each integers pair $\{x, y\}$ encountered in steps 1 and 2 . We also query for the $e$-th root of all prime numbers below $B^{\prime}$.

Step 4 - Descent Initialization. In our game, it is only at this point that the attacker learns the challenge number $t$ whose $e$-th root he must compute.

The descent mimics individual discrete logarithm computations. The descent is initialized by picking a random mask $m$ and two integers $u$ and $v$ such that $\frac{u}{v} \equiv m^{e} t \bmod n$, and which factor simultaneous into primes bounded by $L_{n}\left(\frac{2}{3}, \bullet\right)$.

Step 5 - Descent. We maintain a set $\{(\sigma, \epsilon)\}$ of polynomials $\sigma$ and exponents $\epsilon$ such that $S=\prod \sigma^{\epsilon}$ satisfies:

$$
\begin{aligned}
(S(\alpha)) & =\prod_{\mathfrak{p} \in \mathcal{F}^{\prime}} \mathfrak{p}^{\mu_{\mathfrak{p}}} \cdot I_{1} \quad(\text { algebraic side }), \\
\text { and } \quad \frac{u}{v} S(\rho) & =\prod_{p<B^{\prime}} p^{\nu_{p}} \cdot I_{2} \quad(\text { rational side }) .
\end{aligned}
$$

Initially $S=1$, and the exponents $\nu_{p}$ mark the prime numbers appearing in the factorization of $u$ and $v$.

The remaining terms $I_{1}$ and $I_{2}$ factor into ideals (or primes) outside the factor base. The descent procedure aims at eliminating these ideals. For this purpose, we iteratively use special- $q$ sieving to trade these ideals for ideals of smaller norm.

Using the relations obtained from the factor base extension step, we form another rational fraction $T$ such that the ideal $(S(\alpha) T(\alpha))$ factors into ideals belonging to the smaller factor base $\mathcal{F}$.

Step 6 - Linear Algebra. Once we have reached the point where $I_{1}=(1)$ and $I_{2}=(1)$, we seek a linear combination of the rows of the matrix $M$ which 
equals the valuations and character values corresponding to the algebraic number $S(\alpha) T(\alpha)$.

This inhomogeneous linear system amounts to exhibiting an algebraic number $U(\alpha)$ obtained as a combination of the numbers $x-y \alpha$ found in step 1 , and such that $S(\alpha) T(\alpha) U(\alpha)$ is an $e$-th power in $K$.

Step 7 - End. We use Montgomery's $e$-th root algorithm to write the previous number explicitly as an $e$-th power of an algebraic number $r(\alpha)^{e}$.

By construction, the $e$-th roots of $T(P)$ and $U(P)$ are known by the oracle queries. Using the rational side product form and the corresponding oracle queries, the $e$-th root of $\frac{u}{v} S(P)$ is known as well. We infer:

$$
\sqrt[e]{t}=\frac{1}{m} \cdot \frac{\sqrt[e]{\frac{u}{v} S(P)}}{r(P)} \sqrt[e]{T(P)} \sqrt[e]{U(P)} .
$$

\subsection{Complexity Analysis}

Using the same parameters as in Section [3, all steps except steps 2 to 5 are achieved with complexity $L_{n}\left(\frac{1}{3}, \sqrt[3]{\frac{32}{9}}\right)$.

The complexity of step 2 depends of course on the choice of $\beta^{\prime}$. The summation from $B=L_{n}\left(\frac{1}{3}, \beta\right)$ to $B^{\prime}=L_{n}\left(\frac{1}{3}, \beta^{\prime}\right)$ yields a complexity $L_{n}\left(\frac{1}{3}, \theta\right)$ where $\theta$ ranges from $\sqrt[3]{\frac{32}{9}}$ when $\beta^{\prime}$ is chosen close to $\beta$, up to 1.577 with the suggested value $\beta^{\prime}=\sqrt[3]{\frac{8}{9}}$ (the detailed calculations, omitted for brevity, will be included in the IACR ePrint version of this paper).

The number of oracle queries (step 3$)$ is $L_{n}\left(\frac{1}{3}, \beta^{\prime}=\sqrt[3]{\frac{8}{9}}\right)$.

The descent (steps 4 and 5 ) is analyzed in [5], and found to have complexity $L_{n}\left(\frac{1}{3}, \sqrt[3]{3}\right)$.

We highlight, however, the complexity of the last descent steps, where ideals of norm just above $B^{\prime}=L_{n}\left(\frac{1}{3}, \beta^{\prime}\right)$ have to be canceled. For each such ideal, one relation is sought. Using special- $q$ sieving, we can form $L_{n}\left(\frac{1}{3}, 2 \alpha\right)$ candidates whose algebraic (resp. rational) norm is bounded by $L_{n}\left(\frac{2}{3}, \frac{1}{\delta}+\delta\left(\alpha+\frac{\beta^{\prime}}{2}\right)\right)$ (resp. $\left.L_{n}\left(\frac{2}{3}, \frac{1}{\delta}\right)\right)$. One relation is expected when $\alpha$ satisfies:

$$
2 \alpha-\frac{1}{3}\left(\frac{1}{\delta \beta^{\prime}}+\frac{\delta}{\beta^{\prime}}\left(\alpha+\frac{\beta^{\prime}}{2}\right)+\frac{1}{\delta \beta^{\prime}}\right)=0 .
$$

Substituting $\beta^{\prime}=\sqrt[3]{\frac{8}{9}}$ above, we obtain that the last descent steps are achieved in complexity $L_{n}\left(\frac{1}{3}, 0.99\right)$, which is not dominating. Using $\beta^{\prime}=\beta$ (thereby skipping the factor base extension), this cost would be $L_{n}\left(\frac{1}{3}, 1.27\right)$ which is not dominating either.

This implies that we have some flexibility in the tuning of the factor base extension. In order to match previously completed discrete logarithm 
computations, we chose to extend to $\beta^{\prime}=\sqrt[3]{\frac{8}{9}}$, but this choice should be regarded as unconstrained.

We conclude that the asymptotic complexity of the arbitrary $e$-th root computation is either $L_{n}\left(\frac{1}{3}, \sqrt[3]{\frac{32}{9}}\right)$ or $L_{n}\left(\frac{1}{3}, 1.58\right)$. We believe the latter to be more practical, as is illustrated by our experiments (Appendix B).

\subsection{Computing $e$-th Roots with an AMR-Oracle}

While we have presented and implemented the arbitrary $e$-th root computation algorithm using access to a general $e$-th root oracle, the same can also be achieved using an AMR-oracle only. In this case, the common root $P$ is prescribed, and it is not possible to use a rational side. Nonetheless, the above approach works using two algebraic sides; steps $1,2,6$, and 7 have to be done separately on both sides. Step 4, however, turns out to have a higher complexity requirement $L_{n}\left(\frac{1}{3}, \sqrt[3]{6}\right)$, and the individual descent steps in step 5 are more expensive. We could not demonstrate the practicality of such a setting.

\section{Acknowledgements}

The authors would like to thank P. Zimmermann who provided early experimental input for the quadratic sieve version.

\section{References}

1. Aoki, K., Franke, J., Kleinjung, T., Lenstra, A., Osvik, D.: Electronic newsgroup posting announcing the factorization of the 1039-th Mersenne number by the snfs (May 21, 2007), http://www.loria.fr/zimmerma/records/21039-

2. Bellare, M., Namprempre, C., Pointcheval, D., Semanko, M.: The One-More-RSAInversion Problems and the Security of Chaum's Blind Signature Scheme. Journal of Cryptology 16(3), 185-215 (2003)

3. Buhler, J.P., Lenstra, A.K., Pollard, J.M.: Factoring integers with the number field sieve. In: Lenstra, A.K., Lenstra Jr., H.W. (eds.) The development of the number field sieve. LMN, vol. 1554, pp. 50-94. Springer, Heidelberg (1993)

4. Brier, É., Clavier, C., Coron, J.-S., Naccache, D.: Cryptanalysis of RSA signatures with fixed-pattern padding. In: Kilian, J. (ed.) CRYPTO 2001. LNCS, vol. 2139, pp. 433-439. Springer, Heidelberg (2001)

5. Commeine, A., Semaev, I.: An algorithm to solve the discrete logarithm problem with the number field sieve. In: Yung, M., Dodis, Y., Kiayias, A., Malkin, T.G. (eds.) PKC 2006. LNCS, vol. 3958, pp. 174-190. Springer, Heidelberg (2006)

6. Coron, J.-S., Naccache, D., Stern, J.P.: On the Security of RSA padding. In: Wiener, M.J. (ed.) CRYPTO 1999. LNCS, vol. 1666, pp. 1-18. Springer, Heidelberg (1999)

7. De Jonge, W., Chaum, D.: Attacks on some RSA signatures. In: Williams, H.C. (ed.) CRYPTO 1985. LNCS, vol. 218, pp. 18-27. Springer, Heidelberg (1986) 
8. Eberly, W., Giesbrecht, M., Giorgi, P., Storjohann, A., Villard, G.: Solving sparse rational linear systems. In: Trager, B.M. (ed.) ISSAC 2006, pp. 63-70. ACM Press, New York (2006)

9. Eberly, W., Giesbrecht, M., Giorgi, P., Storjohann, A., Villard, G.: Faster inversion and other black box matrix computations using efficient block projections. In: Brown, C.W. (ed.) ISSAC 2007, pp. 143-150. ACM Press, New York (2007)

10. Girault, M., Misarksy, J.-F.: Selective forgery of RSA signatures using redundancy. In: Fumy, W. (ed.) EUROCRYPT 1997. LNCS, vol. 1233, pp. 495-507. Springer, Heidelberg (1997)

11. Joux, A., Lercier, R.: Improvements to the general number field sieve for discrete logarithms in prime fields. A comparison with the gaussian integer method. Mathematics of Computation 242(72), 953-967 (2003)

12. Lenstra, A.K., Lenstra Jr., H.W., Manasse, M.S., Pollard, J.M.: The number field sieve. In: Lenstra, A.K., Lenstra Jr., H.W. (eds.) AMCP 1998. LNM, vol. 1554, pp. 11-42. Springer, Heidelberg (1993)

13. Lenstra, A.K., Shparlinski, I.: Selective forgery of RSA signatures with fixedpattern padding. In: Proceedings of the 5-th International Workshop on Practice and Theory in Public Key Cryptosystems: Public Key Cryptography. LNCS, vol. 2274, pp. 228-236. Springer, Heidelberg (2002)

14. Misarsky, J.-F.: A multiplicative attack using LLL algorithm on RSA signatures with redundancy. In: Proceedings of Crypto 1997. LNCS, vol. 1294, pp. 221-234. Springer, Heidelberg (1997)

15. Misarsky, J.-F.: How (not) to design RSA signature schemes. In: Imai, H., Zheng, Y. (eds.) PKC 1998. LNCS, vol. 1431, pp. 14-28. Springer, Heidelberg (1998)

16. Montgomery, P.L.: Square roots of products of algebraic numbers. In: W. Gautschi, Ed., Mathematics of Computation 1943-1993: A Half-Century of Computational Mathematics, vol. 48 of Proc. Sympos. Appl. Math., pp. 567-571. AMS (1994)

17. Rivest, R., Shamir, A., Adleman, L.: A method for obtaining digital signatures and public key cryptosystems. CACM 21 (1978)

18. RSA Laboratories, pkcs \#1 : RSA cryptography specifications, version 2.0 (September 1998)

19. Schirokauer, O.: Discrete logarithms and local units. Philos. Trans. Roy. Soc. London Ser. a 345(1676), 409-423 (1993)

\section{A Implementation Details}

As our algorithms are subexponential, the assessment of their experimental behavior is essential. We hence implemented them and actually computed a 512-bit AMR.

We wrote our software chain in $\mathrm{C}$ and $\mathrm{C}++$, relying upon the computer algebra systems PARI-GP and MAGMA for a handful of specific tasks. The attacked instance was $c=10^{154}, e=65,537$ and $n=$ RSA-155 (RSA Laboratories 512-bit challenge modulus).

The polynomial selection (section 3.1) was implemented in MAGMA. To obtain a satisfactory relation yield, we have set $B=2^{22}$ (i.e a factor base comprising 
circa 300,000 prime ideals). For $S=2^{50}$, the polynomial selection program returned the quartic candidate $f(x)=\sum_{i=0}^{4} a_{i} x^{i}$ where:

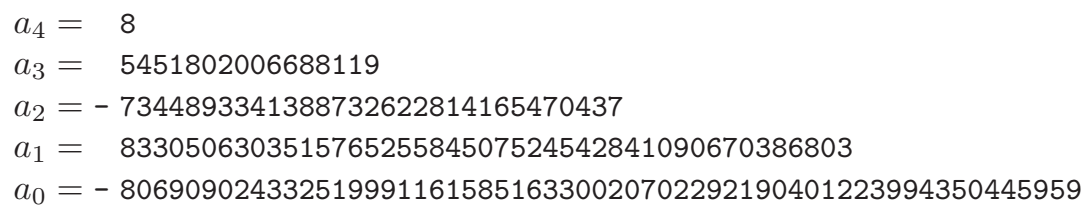

We worked in $K=\mathbb{Q}[x] / f$ and counted 295,842 prime ideals of degree one (or dividing the leading coefficient) in $K$ 's integer ring.

The sieving process was run on a heterogeneous set of CPUs: AMD Opteron 250 at $2.4 \mathrm{GHz}$ and Intel Core- 2 at various clock speeds.

For each special- $q$ ideal written as $\langle q, \alpha-r\rangle$, we isolated the integers $x \in$ $\left[-2^{28}, 2^{28}\right]$ such that the added contribution of factor base ideals to the norm of the ideal $(r+q x-\alpha)$ exceeded $2^{145}$ (out of an order of magnitude just below $\left.2^{200}\right)$. This selection process isolated instantaneously 11 circa 100 candidates of which around nineteen yielded relations. Considering the largest 20,000 ideals in the factor base as special- $q$ ideals, we obtained 380,000 relations. The sieving step was distributed over twenty CPUs and claimed a couple of hours. We stress that we did not use any "large prime" variation.

After pruning the columns corresponding to ideals never encountered in the factorizations, we were left with a row dependency to be obtained on a $283,355 \times 283,355$ matrix. We included four readily computed character columns in the matrix, to ensure that the computed dependency corresponds to an $e$-th power. The dependency was obtained using the block Wiedemann algorithm, with a "blocking factor" of $m=n=8$. This took four $\mathrm{CPU} 4$ hours distributed on four machines to produce one row dependency.

The $e$-th root computation was done in MAGMA.

We started with a product formula $\pi$ whose numerator and denominator had a norm $\approx e^{7.6 \times 10^{5}}$ and with a moderate unit contribution, since the logarithms of the complex embeddings were approximately:

$$
(\lambda+45, \lambda+45, \lambda-155, \lambda+65) \text { where } \lambda=\frac{1}{d} \log \operatorname{Norm}(\pi) \simeq 6710
$$

Here $\lambda$ is the normalizing term. This is quite small since a unit with logarithms of complex embeddings equal to $(45,45,-155,65)$ would correspond to an algebraic integer with coefficients of about twenty decimal digits. The first four reduction steps sufficed to eliminate this unit contribution (i.e. equalling the logarithms of the complex embeddings with their average). After 2,000 reduction steps, we obtained a complete product formula for the root, the remaining $e$-th power being -1 . It took five minutes to compute this $e$-th root.

\footnotetext{
$\overline{10}$ Best amongst a set of 1,000 candidates.

$112.667 \mathrm{GHz}$ Intel Core-2 CPU.

12 2.667 GHz Intel Core-2.
} 
The corresponding final multiplicative dependency involved 242, 700 integers of the form $c+x_{i} \bmod n$.

\section{B Example of an $e$-th Root Computation}

As an experimental illustration of the arbitrary $e$-th root computation, we used once again $n=$ RSA-155. For a public exponent $e=65,537$, we detail the computation of an arbitrary $e$-th root given access to a preliminary $e$-th root oracle (the attacker is given the challenge only once all oracle queries have been performed).

We have chosen a setup resembling a typical NFs factoring experiment or a computation of discrete logarithms. The polynomials $f_{1}=\sum a_{i} x^{i}$ and $f_{2}=$ $\sum b_{i} x^{i}$ are given by the following coefficients, the polynomial $f_{2}$ corresponding to a rational side:

$$
\begin{aligned}
& a_{5}=28200000 \\
& a_{4}=-7229989539851 \\
& a_{3}=-24220733860168568962 \\
& a_{2}=-6401736489600175386662132 \\
& a_{1}=4117850270472750057831223534880 \\
& a_{0}=747474581145576370776244346990929200 \\
& b_{1}=14507315380338583 \\
& b_{0}=-207858336487818193824240150287
\end{aligned}
$$

These two polynomials are easily seen to share a common root $P$ modulo $n$. The sieving stage has been performed only on the number field side. We chose as a small factor base the set of prime ideals of norm below $B=4 \times 10^{6}$ (i.e. 283,042 ideals). For the sieving, we have used the lattice sieving program lasieve4 of J. Franke and T. Kleinjung included in the ggnfs software suite. The program was modified to sieve only on one side. Using a double large prime variation, the sieving step has been completed in two CPU hours on a $2.4 \mathrm{GHz}$ AMD Opteron.

We then extended the factor base to the larger bound $B^{\prime}=2^{32}$. After 44 CPU hours, we were able to relate $37 \%$ of the ideals of this larger factor base to ideals of the smaller factor base (the larger factor base comprises approximately $2 \times 10^{8}$ ideals).

Counting oracle queries related to both sides, we need to perform $4 \times 10^{8}$ queries before being able to compute arbitrary $e$-th roots.

We have implemented the descent procedure using MAGMA, as well as the lasieve4 program, modified in order to account for very large special $q$ 's as used in the descent process. The factorization of the numerous sieve residues produced was handled by the GMP-ECM program.

The descent was initialized on the rational side. We obtained integers $u$ and $v$ which factored into primes with at most 35 decimal digits. Each step of the descent procedure involved a lasieve4 call, in order to select several candidate 
polynomials. Amongst the possible polynomials, our strategy selected the one leading to the fewest ideals outside the factor base (taking into account the large ideals coming from the factor base extension). After 42 descent steps, we obtained a product formula involving 594 prime numbers and ideals below $B^{\prime}=2^{32}$. Some (19) ideals in this product formula belonged to the set of "missed" ideals from the larger factor base. With 21 extra descent steps, these ideals were eliminated. The descent procedure took roughly one hour.

The schedule time for solving the resulting inhomogeneous linear system and computing the algebraic $e$-th root compares in every respect to the data given for the previous example (Appendix A). 